\title{
EDITORIAL
}

\section{QUALITATIVE METHODS IN INFORMATION SYSTEMS RESEARCH}

\author{
Editors of the Special Issue \\ Wasana Bandara (Queensland University of Technology) \\ Walter Fernandez (Australian National University) \\ Bruce Rowlands (Griffith University)
}

Qualitative research methods are widely accepted in Information Systems and multiple approaches have been successfully used in IS qualitative studies over the years. These approaches include narrative analysis, discourse analysis, grounded theory, case study, ethnography and phenomenological analysis. Guided by critical, interpretive and positivist epistemologies (Myers 1997), qualitative methods are continuously growing in importance in our research community.

In this special issue, we adopt Van Maanen's (1979: 520) definition of qualitative research as an umbrella term to cover an "array of interpretive techniques that can describe, decode, translate, and otherwise come to terms with the meaning, not the frequency, of certain more or less naturally occurring phenomena in the social world".

In the call for papers, we stated that the aim of the special issue was to provide a forum within which we can present and debate the significant number of issues, results and questions arising from the pluralistic approach to qualitative research in Information Systems. We recognise both the potential and the challenges that qualitative approaches offers for accessing the different layers and dimensions of a complex and constructed social reality (Orlikowski, 1993). The special issue is also a response to the need to showcase the current state of the art in IS qualitative research and highlight advances and issues encountered in the process of continuous learning that includes questions about its ontology, epistemological tenets, theoretical contributions and practical applications.

In presenting this special issue, we believe that we have succeeded in bringing together a number of high quality contributions representing a broad spectrum of qualitative research, illustrating the wide range of interesting work being done by qualitative IS researchers; from ethnography, story-telling, theorising, generalising etc. Some papers attempt to better position different/ new forms of qualitative research and their evolution, and some others show how qualitative research can beapplied for empirically supported outcomes.

The first paper by Shah J Miah, Justin Debuse, and Don Kerr introduces a development-oriented approach to evaluate system design quality in the context of decision support systems (DSS). Following a design science paradigm, the article describes a study that investigated the applicability of the 'six-stages' model presented by Peffers et al. (2008) for evaluation of DSS. Using a sociotechnical lens, the authors successfully captured and analysed the views of different stakeholders regarding the six design stages of define problem, define objectives, design and development, demonstration, evaluation and communication. The study proposes an approach that can serve to evaluate whether the entire development and design cycle meets the requirements for DSS development. The approach advanced in this paper is able to capture the artefact's development process as well as its context, thus, allowing the evaluation of the artefact within an organisation.

The second paper by Goran Goldkuhl presents an alternative research approach; 'Practice research'. Goldkuhl presents how the proposed research approach, is founded in pragmatism, describing how it can be useful to contribute to general practice through abstract and useful knowledge and to study the empirical field as 'interconnected practices'. Practice research is presented here as a novel means to 
address some of the controversies and confusions observed in traditional Action research (AR) approaches. Two case examples are presented to further illustrate the notion and application of 'Practice research'.

The third paper by Matthias Goeken and René Börner attempts to address one of the most prominent issues in IS qualitative research; generalization. First, they provide a detailed overview of existing approaches to generalizations and its outcomes (various means of conceptualizations), providing a thorough overview of the current status of these topics as observed in the current literature. They then extend the notion of generalization by discussing how abstraction mechanisms of conceptual modelling, can be applied in conjunction with existing approaches in the realm of qualitative information systems research, for generalization purposes. The authors illustrate the application of the suggested generalisation approach through an explorative single case study.

The fourth paper by France Belanger brings to this special issue a timely discussion on focus groups as a research method. Belanger reviews the IS literature to present a thorough discussion on the potential contribution of focus groups to IS theorising. The article contributes to clarify important issues related to focus group research and shows how the approach can be used to explore, to explain and to validate theory; both as a sole method or in combination with other research methods. Belanger's expertise with the method and the manner in which the argument is provided offer valuable information and opens the door to the many possibilities focus groups has to offer to our field.

The fifth paper, by Stefan Thalman and Ronald Maier propose a procedure for the study of knowledge work practices adapted from collaborative ethnography that can be used to inform requirements gathering, and the design of information systems. Their procedure is illustrated with an example of a collaborative ethnographic study involving seven organisations in four European countries who took part in a large-scale international IS research and development project. The authors critically discuss the procedure's implementation and limitations.

The sixth paper, by Julie Kendall and Kenneth Kendall review how early information systems researchers may have used fragments of stories as an alternative method to gathering data for the design, development, or documentation of information systems. From a theoretical perspective, informed by the use of social construction of technology theory, the authors develop an innovative research method based on storytelling, aiming to inspire IS researchers to realise the depth and richness of myths found in organizational stories, and to appreciate and use stories in their own work. The paper is instructive in that it describes how storytelling differs from other qualitative approaches, and offers an exemplary example of how their research observed the principles of Klein \& Myers' (1999) principles for conducting interpretive research.

The interest that the call for papers generated and the high number of submissions received afforded us a privileged opportunity for working with a variety of authors and topics toward furthering qualitative IT/IS research. Though only a small portion of the submissions made their way into this special issue, we were heartened by the authors' enthusiastic response and by the quality of all the works submitted. We look forward to the continuing contribution of qualitative research in the forthcoming issues of AJIS.

Finally, we hope you enjoy reading these papers as we did. Special thanks must go to our reviewers for this special issue, for their time and hard work in evaluating and discussing papers and all the constructive feedback provided to assist the authors improve their papers. 


\section{REFERENCES}

Klein, H. K. \& Myers, M. D. (1999) A Set of Principles for Conducting and Evaluating Interpretive Field Studies in Information Systems, MIS Quarterly, Special Issue on Intensive Research, (23:1), pp 67-93.

Myers, M.D. (1997) Qualitative Research in Information Systems, MIS Quarterly (21:2), pp 241-242.

Orlikowski, W.J. (1993) CASE tools are organizational change: Investigating Incremental and Radical Changes in Systems Development, MIS Quarterly (17:3), pp 309-340.

Peffers, K., Tuunanen, T., Rothenberger, M.A. and Chatterjeea, S. (2008) Design Science Research Methodology for IS Research, Journal of Management Information Systems (24:3), pp 45-77.

Van Maanen, J. (1979) Reclaiming qualitative methods for organizational research: A preface. Administrative Science Quarterly (24:4), pp 520-526.

\section{ABOUT THE AUTHORS}

Wasana Bandara is a Senior Lecturer in Information Systems (IS) at the Information Systems School, Science and Engineering Faculty of the Queensland University of Technology, Brisbane, Australia. Wasana received her PhD from Queensland University of Technology, in 2007. She is author/co-author of over 50 refereed publications, which are predominantly qualitative. Her research areas are on IS research methods, Business Process Management, ICT enabled services, and process improvements in emerging economies. Wasana has been an IS/ BPM educator for twelve years and she has been conducting training on qualitative research to post graduate IS students. Wasana was the co-chair of the QualIT 2010 conference, hosted jointly in conjunction with ACIS2010.

Walter Fernandez is an Associate Professor at the Research School of Accounting and Business Information Systems, the Australian National University. Walter's research interest includes: management of major projects, ICT-enabled organizational change and modernization, and qualitative research methods. Walter is the Inaugural Chair of the Association of Information Systems Special Interest Group on Grounded Theory Methodology, has published many journal and conference articles, conducted qualitative research seminars in more than ten countries, serves in several international journals as reviewer, associate editor and senior editor, and is a past co-chair of QualIT 2010 .

Bruce Rowlands is a Senior Lecturer in Information Systems within the School of ICT at Griffith University, Brisbane. Bruce received his PhD from The University of Technology, Sydney. Bruce has published qualitative research in journals including EJIS, IT\&P, SJIS, AJIS; and has presented his work at ICIS. Bruce is a past co-chair of the QualIT conference hosted initially by Griffith University in 2004, 2005, 2006; and co-chair 2010 presented in conjunction with ACIS2010. His research interests broadly focus on the human, organisational, and institutional aspects of systems development. His research is informed by the social informatics literature as a means for conceptualising the social context of information systems development. 